Rafaela Maria Fiqueiredo da Costa? O- https://orcid.org/0000-0002-4323-5585

Ângela Maria Mendes Abreu² Ohttp://orcid.org/0000-0002-7894-4242

Luciana Fernandes Portela' Ohttps://orcid.org/0000-0001-8961-468X

Gunnar Glauco de Cunto Carelli

Taets $^{3}$

Ohttps://orcid.org/0000-0003-4427-7864

Rafael Tavares Jomar'

(2) htpp://orcid org/0000-0002-4101-7138

\section{Associação entre horas de trabalho e de descanso e o uso de substâncias psicoativas entre motoristas profissionais de caminhão}

\author{
Association between hours of work and rest and the use of \\ psychoactive substances among professional truck drivers
}

D0I: 10.1590/0047-2085000000353

\begin{abstract}
RESUMO
Objetivo: Investigar a associação de horas diárias de trabalho e de descanso com o uso de substâncias psicoativas entre motoristas profissionais de caminhão. Métodos: Estudo transversal conduzido em 2016 com 354 motoristas profissionais de caminhão que aguardavam a inspeção da mercadoria transportada em um Posto de Controle Fiscal localizado na cidade de Itatiaia, RJ, Brasil. A associação entre horas diárias de trabalho (exposição), horas diárias de descanso (exposição) e uso de substâncias psicoativas (desfecho) foi investigada por meio de modelos de regressão linear que estimaram coeficientes de regressão $(\beta)$ e respectivos erros-padrão, considerando $p<0,05$. Resultados: Mostraram-se positivas as associações entre horas diárias de trabalho e uso de anfetamina ( $\beta=0,91$; erro-padrão = $0,19 ; p<0,01)$ e de cocaína/crack $(\beta=1,32$; erro-padrão $=0,35 ; p<0,01)$ e negativa a associação entre horas diárias de descanso e uso de anfetamina $(\beta=-0,43$; erro-padrão $=0,09 ; p<0,01)$. Conclusão: $\mathrm{Ho}_{\mathrm{O}}$ ras diárias de trabalho e de descanso parecem ser determinantes do uso de anfetamina e de cocaína/ crack entre motoristas profissionais de caminhão.
\end{abstract}

PALAVRAS-CHAVE

Jornada de trabalho, consumo de bebidas alcoólicas, drogas ilícitas, veículos automotores, riscos ocupacionais.

\section{ABSTRACT}

Objective: To investigate the association of hours of work and rest with use of psychoactive substances among professional truck drivers. Methods: Cross-sectional study conducted in 2016 with 354 professional truck drivers waiting for the inspection of the goods transported at a Fiscal Control Post located in the city of Itatiaia, RJ, Brazil. The association between daily working hours (exposure), daily rest hours (exposure) and use of psychoactive substances (outcome) was investigated using linear regression models that estimated regression coefficients $(\beta)$ and respective standard error, considering $p<0.05$. Results: They were positive the associations between daily working hours and use of amphetamine $(\beta=0.91$; standard error $=0.19 ; p<0.01)$ and cocaine $/$ crack $(\beta=1.32$; standard error $=0.35$; $p<0.01)$ and the association between daily rest hours and use of amphetamine $(\beta=-0.43$; standard error $=0.09 ; p<0.01)$ was negative. Conclusion: Daily hours of work and rest seem to be determinants of the use of amphetamine and cocaine/crack among professional truck drivers.

\section{KEYWORDS}

Work hours, alcohol drinking, illicit drugs, trucks, occupational risks. 


\section{INTRODUÇÃO}

Motoristas profissionais de caminhão costumam usar substâncias psicoativas para combater a fadiga e a sonolência em viagens de longa distância ${ }^{1-4}$. O excesso de horas de trabalho e o pouco tempo para descanso desses motoristas decorrem da extenuante responsabilidade no cumprimento de prazos e horários de entrega das mercadorias estabelecidos pelas empresas contratantes/empregadoras ${ }^{5,6}$.

Considerando que essas condições de trabalho podem tornar motoristas profissionais de caminhão vulneráveis ao uso de substâncias psicoativas ${ }^{1-4}$, o objetivo deste estudo foi investigar a associação de horas diárias de trabalho e de descanso com o uso de substâncias psicoativas por essa população.

\section{MÉTODOS}

Trata-se de um estudo transversal conduzido entre os meses de janeiro e junho de 2016 no Posto de Controle Fiscal de Nhangapi, que pertence à Secretaria de Estado de Fazenda do Rio de Janeiro e está localizado no Km 324 da Rodovia Presidente Dutra na cidade de Itatiaia, RJ, Brasil. Essa rodovia liga as duas maiores cidades do país e nela trafegam diariamente cerca de 5 mil veículos de transporte de carga.

Ao passar pelo Posto de Controle Fiscal, qualquer motorista de veículo de transporte de carga deve apresentar a nota fiscal da mercadoria transportada para averiguação, bem como uma eventual inspeção da mercadoria. De terça à quinta-feira nos turnos da manhã, tarde ou noite, enquanto aguardavam a inspeção da mercadoria transportada, 354 motoristas profissionais de caminhão que atendiam aos critérios de inclusão (possuir Carteira Nacional de Habilitação de categoria $\mathrm{C}$ ou E) e de exclusão (ter sido detido pela Polícia Militar no Posto de Controle Fiscal) do estudo foram abordados de forma não aleatória por entrevistadoras devidamente treinadas, que, após a exposição da natureza e dos objetivos dele, fizeram o convite para participação. Cabe esclarecer que, por questões logísticas, as entrevistadoras se revezaram durante os seis meses de coleta de dados, que, por sua vez, não ocorreu em todas as terças, quartas e quintas-feiras do período, tampouco em todos os turnos de um mesmo dia de entrevistas.

As entrevistas, cujo tempo de duração oscilou entre 30 e 40 minutos, foram conduzidas em local reservado do Posto de Controle Fiscal, utilizando-se um questionário multidimensional composto por questões sociodemográficas e outras relacionadas à saúde, ao estilo de vida e à profissão de caminhoneiro. As horas diárias de trabalho foram medidas a partir da seguinte pergunta: "Em geral, quantas horas no total o senhor trabalha por dia?". As horas diárias de descanso, por sua vez, foram medidas por meio da pergunta "Quantas horas por dia o senhor descansa?". Já o uso de substâncias psicoativas foi avaliado pela seguinte questão: "Durante os três últimos meses, com que frequência você utilizou bebidas alcoólicas, anfetamina (rebite) ou cocaína/crack?", e as opções de resposta e pontuações correspondentes para cada uma dessas substâncias eram "nunca" (0), "uma ou duas vezes" (3), "mensalmente" (4), "semanalmente" (5) ou "diariamente ou quase todos os dias" (6). Outras covariáveis também foram medidas, quais sejam: idade (em anos), renda per capita familiar (em salários-mínimos), trabalho noturno (sim ou não), tipo de carga transportada (viva, seca/não perecível, perecível, de veículos, de valor ou de produtos perigosos) e tempo médio de duração de viagem a trabalho (em dias). A renda per capita familiar foi calculada dividindo-se o resultado do somatório da renda mensal de cada pessoa que residia com o motorista, inclusive a do próprio, pelo número total de residentes e transformada, na sequência, em salários mínimos, que era igual a $\mathrm{R} \$ 788,00$ no período de coleta de dados.

A associação entre horas diárias de trabalho (exposição), horas diárias de descanso (exposição) e uso de substâncias psicoativas (desfecho) foi investigada por meio de modelos de regressão linear que estimaram coeficientes de regressão ( $\beta$ ) e respectivos erros-padrão. As análises estatísticas, conduzidas no software SPSS 23.0 para cada exposição e tipo de substância separadamente, foram ajustadas por idade, renda per capita familiar, trabalho noturno, tipo de carga transportada e tempo médio de duração de viagem a trabalho, considerando $p<0,05$. Os $\beta$ ajustados representaram, portanto, a mudança média na pontuação correspondente à frequência de uso de cada substância psicoativa em função do aumento de uma hora de trabalho ou de descanso. Vale sublinhar que a distribuição normal dos erros foi testada e confirmada, o que permitiu o uso de modelos de regressão linear.

A condução do estudo foi aprovada pelo Comitê de Ética em Pesquisa da Escola de Enfermagem Anna Nery/ Hospital Escola São Francisco de Assis da Universidade Federal do Rio de Janeiro em 16 de dezembro de 2015 (CAAE: 51708715.5.0000.5238; Parecer: 1.370.081). Todos os participantes assinaram um termo de consentimento livre e esclarecido concordando em fazer parte dele.

\section{RESULTADOS}

Como não houve recusas, os 354 motoristas profissionais de caminhão abordados aceitaram participar do estudo. Todos eram do sexo masculino, com média de idade igual a 42,0 anos $( \pm 9,6) ; 53,7 \%$ trabalhavam no turno da noite, $79,1 \%$ tinham renda per capita familiar menor que um salário mínimo e 45,8\% transportavam carga seca/não perecível. O tempo médio de duração de viagem a trabalho era de 3,7 dias $( \pm 3,5)$, a média de horas diárias de trabalho era de $13,0( \pm 3,4)$ 
e a média de horas diárias de descanso, de 6,3 ( $\pm 1,7)$. No tocante ao uso de substâncias psicoativas (Tabela 1), 14,3\% dos motoristas relataram uso de bebidas alcoólicas, 2,6\% de anfetamina e 0,9\% de cocaína/crack pelo menos uma vez por semana.

A associação entre horas diárias de trabalho e uso de anfetamina e de cocaína/crack mostrou-se positiva $(p<0,01)$, já a associação entre horas diárias de descanso e uso de anfetamina mostrou-se negativa $(p<0,01)$. Horas diárias de trabaIho e horas diárias de descanso, contudo, não se mostraram associadas ao uso de bebidas alcoólicas ( $p>0,30$ ) (Tabela 2).

\section{DISCUSSÃO}

Horas diárias de trabalho e horas diárias de descanso não se mostraram associadas apenas ao uso de bebidas alcoólicas, que, cabe ressaltar, é a substância psicoativa mais usada por motoristas profissionais de caminhão em todo o mundo'. Como desde 2008 vigora no Brasil uma lei que proíbe o consumo de álcool por condutor de veículo automotor, motoristas profissionais de caminhão que diariamente trabalham muitas horas e descansam outras poucas parecem não ter muitas oportunidades para consumir essa substância durante as viagens de trabalho, pois, se flagrados dirigindo sob efeito dela, estariam sujeitos a penalidades como prisão, multa suspensão do direito de dirigir e apreensão do veícu$\mathrm{IO}^{7}$, o que prejudicaria seu exercício profissional. O fato de o uso de bebidas alcoólicas ser socialmente aceito e culturalmente estimulado também ajuda a explicar por que a associação entre esses fenômenos não foi aqui confirmada, pois a frequência média do uso dessa substância entre motoristas profissionais de caminhão é igual a 54,3\%, segundo revisão sistemática ${ }^{1}$. No entanto, existem evidências de que o uso de bebidas alcoólicas é preditor do uso de anfetamina por essa população ${ }^{3}$.

Concordante com os resultados do estudo em tela, Oliveira et al. ${ }^{3}$, que testaram se condições ocupacionais de motoristas profissionais de caminhão estavam associadas ao uso de anfetamina, evidenciaram que trabalhar mais de 12 horas diárias aumentava em pouco mais de duas vezes a probabilidade de usar anfetamina. Sinagawa et al.2. que investigaram a associação entre uso de substâncias psicoativas e duração de viagem a trabalho, estimaram probabilidade quase 10 vezes maior de uso de anfetamina entre motoristas de caminhão que percorriam distâncias superiores a 270 km. Conduzidas por Bombana et al. ${ }^{4}$, análises de amostras de fluido oral de motoristas profissionais de caminhão no estado de São Paulo, Brasil, mostraram que a prevalência do uso de anfetamina era maior entre aqueles que percorriam distâncias superiores a $1.500 \mathrm{~km}$.

Assim, os resultados deste e dos estudos anteriormente citados parecem corroborar o uso de anfetamina por motoristas profissionais de caminhão para combater a fadiga e a sonolência em viagens de longa distância e/ou duração.

Além disso, vale destacar que a hipótese de que é maior a frequência do uso de substâncias psicoativas por essa população, quanto menos horas ela descansar, foi aqui confirmada por meio da associação negativa entre horas diárias de descanso e uso de anfetamina. Tal associação pode ser explicada por causa do efeito dose-resposta da anfetamina no sistema nervoso central, que, ao reduzir a sensação de fadiga e disfarçar o cansaço, mantém o usuário acordado ${ }^{8}$ e, por consequência, desfrutando de menos horas de descanso.

O presente estudo também evidenciou associação positiva entre horas diárias de trabalho e uso de cocaína/crack entre motoristas profissionais de caminhão, embora pesquisas brasileiras prévias não tenham encontrado associação

Tabela 1. Uso de substâncias psicoativas entre motoristas profissionais de caminhão - Itatiaia, Brasil, 2016 (N = 354)

\begin{tabular}{|c|c|c|c|c|c|c|}
\hline \multirow{2}{*}{ Frequência } & \multicolumn{2}{|c|}{ Bebidas alcoólicas } & \multicolumn{2}{|c|}{ Anfetamina } & \multicolumn{2}{|c|}{ Cocaína/crack } \\
\hline & $n$ & $\%$ & $\mathbf{n}$ & $\%$ & $n$ & $\%$ \\
\hline Nunca & 228 & 64,4 & 328 & 92,6 & 348 & 98,2 \\
\hline Uma ou duas vezes & 39 & 11,0 & 06 & 1,7 & 01 & 0,3 \\
\hline Mensalmente & 36 & 10,2 & 11 & 3,1 & 02 & 0,6 \\
\hline Semanalmente & 46 & 12,9 & 07 & 2,0 & 02 & 0,6 \\
\hline Diariamente ou quase todos os dias & 05 & 1,4 & 02 & 0,6 & 01 & 0,3 \\
\hline
\end{tabular}

Tabela 2. Associação de horas diárias de trabalho e de descanso com uso de substâncias psicoativas entre motoristas profissionais de caminhão - Itatiaia, RJ, Brasil, 2016

\begin{tabular}{|c|c|c|c|c|c|c|}
\hline \multirow{2}{*}{ Jornada de trabalho } & \multicolumn{2}{|c|}{ Bebidas alcoólicas } & \multicolumn{2}{|c|}{ Anfetamina } & \multicolumn{2}{|c|}{ Cocaína/crack } \\
\hline & $\beta$ (EP) & $\mathbf{p}$ & $\beta$ (EP) & $\mathbf{p}$ & $\beta$ (EP) & $\mathbf{p}$ \\
\hline Horas diárias de trabalho & $-0,06(0,99)$ & 0,55 & $0,91(0,19)$ & $<0,01$ & $1,32(0,35)$ & $<0,01$ \\
\hline Horas diárias de descanso & $0,05(0,05)$ & 0,32 & $-0,43(0,09)$ & $<0,01$ & $-0,26(0,17)$ & 0,13 \\
\hline
\end{tabular}

ß: coeficiente de regressão; EP: erro-padrão. 
entre uso de cocaína e distância percorrida em viagens a trabalho ${ }^{2,4}$, uma variável proxy de seu tempo de duração. Explicação razoável para resultados tão distintos é o fato de tais pesquisas terem sido conduzidas até 2014, ano em que foi proibida a comercialização de inibidores de apetite feitos à base de anfetamina no Brasil, o que parece ter feito com que motoristas profissionais de caminhão adotassem, a partir de então, outras substâncias como substitutas à anfetamina, como a cocaína ${ }^{3,4}$, embora seus efeitos estimulantes sejam mais curtos que os da anfetamina ${ }^{4}$.

Aponta-se como limitação do estudo o fato de a amostra ser não probabilística, o que prejudica a generalização de seus resultados, e de a coleta de dados ter sido feita por meio de entrevista, o que pode ter levado alguns motoristas a negarem o uso de substâncias psicoativas por constrangimento (viés de prevaricação), mesmo que as entrevistadoras tenham garantido o sigilo e o anonimato quanto às informações prestadas. Outra limitação que merece destaque refere-se ao desfecho, que não foi mensurado por meio de um instrumento validado; por esse motivo, é prudente considerar a ocorrência de viés de informação (viés de instrumento), o que pode ter afetado a magnitude das associações encontradas, mas não a direção delas, uma vez que estão em consonância com a literatura.

Em contrapartida, a evidência de associação entre horas diárias de trabalho e uso de cocaína/crack pode ser apontada como um ponto forte do estudo, pois ele é o primeiro a suscitar a hipótese de que a cocaína/crack pode estar substituindo a anfetamina no combate à fadiga e à sonolência de motoristas profissionais de caminhão desde 2014, ano em que foi proibida no Brasil a comercialização de inibidores de apetite feitos à base de anfetamina. Vale destacar que a substituição de anfetamina por cocaína/crack por essa população é apenas uma hipótese, pois somente 1,8\% dos entrevistados afirmaram ter usado cocaína/crack. Pesquisas futuras com amostras maiores e métodos de coleta de dados capazes de minimizar viés de prevaricação são fundamentais para o melhor entendimento desse fenômeno.

Ainda que seu desenho transversal limite a avaliação da relação causal entre horas diárias de trabalho e de descanso e uso de substâncias psicoativas, o estudo aponta para a importância de as autoridades competentes considerarem que a jornada de trabalho - aqui representada pelas horas diárias de trabalho e de descanso - parece ser determinante do uso de anfetamina e de cocaína/crack em motoristas profissionais de caminhão. Alguns pontos importantes e cabíveis de serem observados por empregadores e motoristas profissionais de caminhão no combate à fadiga e à sonolência decorrentes da intensa jornada de trabalho já haviam sido apontados por um trabalho anterior, quais sejam: redução do tempo de direção veicular, aumento do número de pausas para descanso durante o trabalho e programação de cochilos durante o dia e de descanso durante a noite (sono noturno) para manter a sincronização dos ritmos biológicos ${ }^{5}$.

\section{CONCLUSÃO}

O estudo evidenciou associação positiva entre horas diárias de trabalho e uso de anfetamina e de cocaína/crack e associação negativa entre horas diárias de descanso e uso de anfetamina, apontando para a importância de as autoridades competentes considerarem que a jornada de trabalho parece ser determinante do uso de anfetamina e de cocaína/ crack por motoristas profissionais de caminhão.

\section{CONTRIBUIÇÕES INDIVIDUAIS}

Rafaela Maria Figueiredo da Costa, Ângela Maria
Mendes Abreu, Luciana Fernandes Portela e Gunnar
Glauco de Cunto Carelli Taets - Contribuíram na concepção e desenho do estudo e na revisão crítica do conteúdo intelectual do artigo.

Rafael Tavares Jomar - Contribuiu na concepção e desenho do estudo, na análise de dados e na redação do artigo. Todos os autores aprovaram a versão final a ser publicada.

\section{CONFLITOS DE INTERESSES}

Os autores declaram não haver conflitos de interesses.

\section{REFERÊNCIAS}

1. Girotto E, Mesas AE, De Andrade SM, Birolim MM. Psychoactive substance use by truck drivers: a systematic review. Occup Environ Med. 2014;71(1):71-6.

2. Sinagawa DM, De Carvalho HB, Andreuccetti G, Do Prado NV, De Oliveira KC, Yonamine M, et al. Association between travel length and drug use among Brazilian truck drivers. Traffic Inj Prev. 2015;16(1):5-9.

3. Oliveira LG, Souza LMA, Barroso LP, Gouvêa MJC, Almeida CVD, Muñoz DR, et al. Occupational conditions and the risk of the use of amphetamines by truck drivers. Rev Saúde Pública. 2015;49:61.

4. Bombana HS, Gjerde H, Dos Santos MF, Jamt REG, Yonamine M, Rohlfs HJC, et al. Prevalence of drugs in oral fluid from truck drivers in Brazilian highways. Forensic Sci Int. 2017;273:140-43.

5. Narciso FV, De Mello MT. Safety and health of professional drivers who drive on Brazilian highways. Rev Saúde Pública. 2017;51:26.

6. Dini G, Bragazzi NL, Montecucco A, Rahmani A, Durando P. Psychoactive drug consumption among truck-drivers: a systematic review of the literature with meta-analysis and metaregression. J Prev Med Hyg. 2019;60(2):E124-39.

7. Brasil. Lei no 11.705 , de 19 de junho de 2008. Altera a Lei no 9.503, de 23 de setembro de 1997, que institui o Código de Trânsito Brasileiro. Diário Oficial da União, Brasília, p. 33, 20 jun. 2008. Seção 1.

8. Musshoff F, Madea B. Driving under the influence of amphetamine-like drugs. J Forensic Sci. 2012;57(2):413-19. 